\title{
New Turning-tilting Bed and Head-traction Unit
}

Sir Ludwig GuttmanN, of the National Spinal Injuries Centre, Stoke Mandeville Hospital, Aylesbury, Bucks, writes: The care and management of any seriously ill patient after injury or disease places heavy demands on both nursing and medical staff if complications from recumbency, such as pressure sores, lung complications, and stasis in the urinary tract leading to ascending infection and stone formation, are to be avoided. People paralysed as a result of fractures or fracture-dislocations of the spine have always been particularly prone to these complications on account of their bladder paralysis and the initial loss of vasomotor control and tone of all tissues in the paralysed area, resulting in the lowering of tissue resistance to pressure. It is true that these complications can be avoided by the employment of pillows or sorbo-rubber packs, combined with regular two-hourly turning day and night from the supine to the lateral position. However, four persons are necessary, especially in the early stages after paraplegia or tetraplegia, to carry this out properly, and the strain on the nursing staff is only too obvious. Because of the high survival rate of traumatic paraplegics and tetraplegics, even those associated with severe injuries to the chest and other parts of the body, on the one hand, and the increasing difficulty in obtaining adequate nursing staff on the other, turningbeds have been introduced. The best known is the Stryker frame and its modifications. However, this type of turning-frame has its disadvantages in the management of fracturedislocations, especially those of the thoracic and lumbar vertebrae. While in such a frame, with rolls placed underneath the fracture to reduce the fracture-dislocation, hyperextension by posture can be achieved in the supine position, this cannot be maintained when turning the patient on to the abdominal position, which is the only alternative in this type of turning-bed. Thus the object of maintaining hyperextension to secure realignment and promote stability of the broken spine is defeated. Moreover, in traumatic paraplegics with associated fractures of the ribs, pelvis, or long bones, and in particular patients with haemothorax or pneumothorax (let alone those who are unconscious), turning on to the abdominal position is clearly too hazardous and usually contraindicated. If such patients have to maintain their recumbency in the supine position day and night, owing to lack of regular turning from the supine to the lateral position, early development of pressure sores is inevitable.

For some time I have been engaged in the development of an electrically operated turning-bed on which hyperextension of traumatic paraplegics could be maintained while the patients were turned from the supine to the lateral position in the same way as it is done manually. This aim has now been achieved since Egerton Engineering Limited succeeded in designing such a bed (A. C. Egerton Engineering Ltd., Tower Hill, Horsham, Sussex).

Two types of bed have been constructed: one which allows turning only from the supine to the lateral position; the other which, in addition to this turning, can also be tilted up and down. Both types have had extensive trials in this centre, and it has been proved that postural reduction of fracturedislocations of even the most severe type can be maintained safely during the turning procedure. Moreover, permanent recumbency in the supine position is avoided, and thus stagnation in the urinary tract as well as the development of pressure sores can be prevented.

The following details of the construction of the Egerton/Stoke Mandeville turningand-tilting bed demonstrate the management of a traumatic tetraplegic patient. The bed is constructed in mild steel tubing, and the tubes are electrically welded. The height of the bed (to top of mattress) is $30 \frac{1}{4}$ in. $(77 \mathrm{~cm}$.), the length of the bed-frame is $92 \frac{1}{2}$ in. (235 cm.), and the width is $45 \frac{1}{2}$ in. $(116 \mathrm{~cm}$.). The bed is mounted on 7-in. $(18-\mathrm{cm}$.) castors, which have brakes, and the head and foot rails are detachable, giving easy access to the patient. The bed is constructed in such a way that it can easily be broken down for shipping quantities of beds, and at its destination easily reassembled.

The bed is provided with a three-sectional polyester mattress of $\mathrm{G} 14$ density. The mattress platform is divided longitudinally into two parts, each part two-thirds of the bed's width, both parts being hinged at the top. This allows movement of the patient to take place without compressing the surface of the mat-

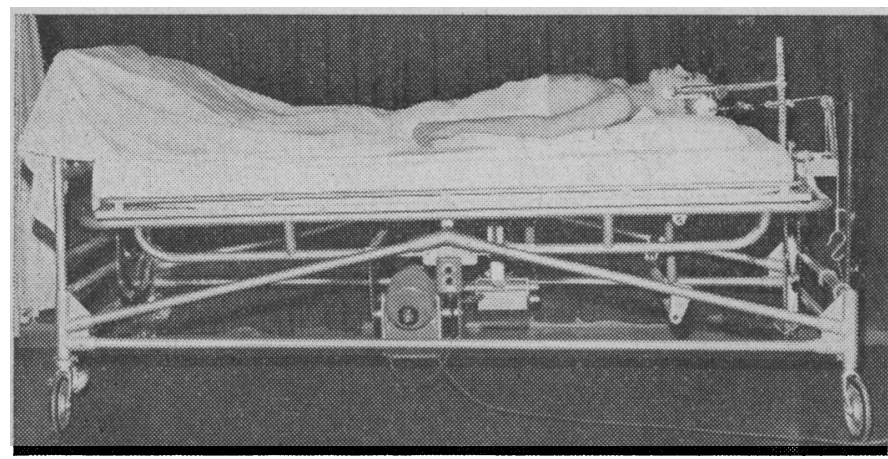

FIG. 1 tress, and thus avoids creases on the patient's body. Symmetry is retained on the bed by a low tubular rail, which makes it impossible for the three sectional parts of the mattress to overlap at any point.

The turning of the bed is accomplished by the action of two small single-phase electric motors fixed on either side of the bed and connected with a threaded shaft. Each motor has its own switch-box, which operates the motor facing the attendant. By pushing the button, each side of the bed is lifted, and thus the patient can be turned from the supine position to a 70 degrees lateral position in a few seconds (Figs. 1 and 2). Adjustments to the switch-box are possible to allow patients in later stages of paraplegia or tetraplegia, especially those who have been provided with a turning-bed at home, to carry out the turning of the bed themselves by operating a switch from the bed, either by hand or, as in the case of high cervical lesions with paralysis of all muscles of the upper limbs, by mouth with the aid of a tube. As the motors are independent it is possible, during turning, to raise slightly the side of the bed on which the patient rests. This eliminates any possibility of slipping, and gives the patient an added feeling of security. Furthermore, the patient can be tilted manually by a handle, head and feet up or down, to a maximum angle of 14 degrees (Fig. 3). No matter to what angle or how often patients are turned, they always return to the centre of the bed. The tilting mechanism can, of course, if so desired, be operated electrically instead of manually.

\section{GutTMANN HeAD-TRACTION UNIT}

For the treatment of traumatic tetraplegics after fracture-dislocation of the cervical spine, both the turning and turning-tilting bed can be provided, as can any other hospital bed, with any type of skull calliper described by Crutchfield, Blackburn, Cone, and others. However, it must be remembered that, what-

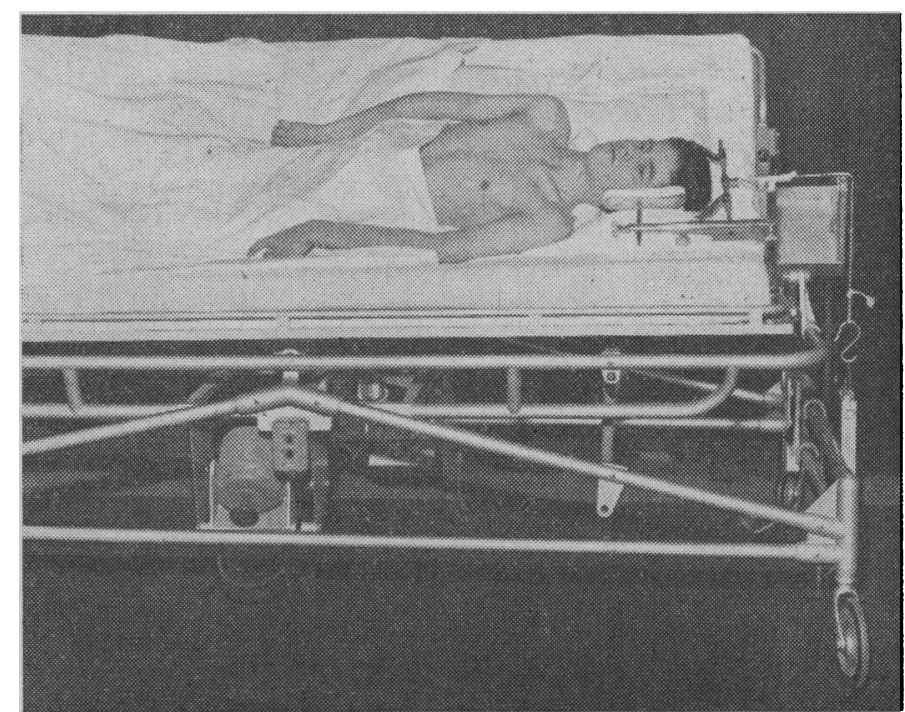

FIG. 2 


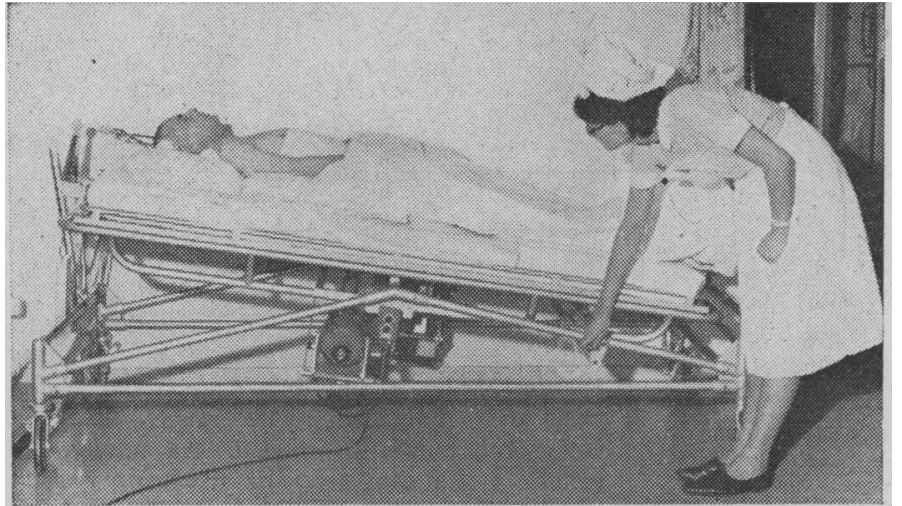

FIG. 3

ever the type of bed used for skull traction in the treatment of the broken cervical spine, it is necessary to disconnect the traction cord of the pulley and weights when turning the patient from the supine to the lateral position. Consequently, the person who has the task of holding the skull calliper in extension and traction has at the same time to support the head during the turning procedure. Therefore greatest care has to be exercised to keep the head in line with the axis through the length of the body in order to prevent any torsion of the neck which could result in redislocation of the broken spine. Furthermore, the person who is holding the head must delay reconnexion of the traction cord to the weight until all arrangements have been made to secure the proper lateral positioning of the head on special pillow supports, in line with the axis of the body. All this can now be avoided by the employment of a special head-traction unit which has been designed to my instructions primarily for use with either of the Egerton/Stoke Mandeville beds. The head-traction unit will allow the patient to be turned from side to side at frequent intervals without any discontinuation of the skull-traction cord ; the head will remain in line with the axis through the length of the body, and correct traction will be maintained during the process of turning.
The new device consists of: (1) a tray which is placed under the central section of the three-sectional mattress ; (2) two long square pillars to which are attached the stems that carry the face pads; (3) the central pulley set, which can be raised or lowered; and (4) the container for the weights. Fig. 4 shows these details.

This equipment has been designed to simplify the procedure of turning these patients and at the same time ensure efficiency so that the turning can be done quickly by the nursing staff or ward orderlies. To prepare the head-traction unit for use the tray is placed underneath the central section of the three-sectional mattress and the long square pillars to which are attached the stems which carry the face pads. The patient is then placed in position, and the central pulley is raised or lowered by means of the knurled wheel directly beneath it to bring it into direct line with the traction cord of the calliper, which is then passed over the pulley and wound round and through the spiral attachment below the knurled wheel. The container for the weights is then attached to the cord, and the appropriate weights are inserted.

The height of the arms which carry the face-pads attachment can be adjusted to suit each individual patient. When this is completed the patient's head is held firmly but not harshly in position, and he can be turned from the supine to the lateral position lying continuously with his head on the underlying face-pad. The correct traction can be maintained, no matter what degree, from 0 to
70 degrees. Fig. 5 shows the head-traction unit in action with the patient turned laterally. For attending the patient's back for washing, etc., the head-traction cord and weight can be disconnected from the central pulley and connected with a hook fixed on a detachable metal rod. The turning as well as the turning-tilting bed can, of course, also be used for the management of patients with other forms of severe disability or disease, such as high polio cases, rheumatoid arthritis, strokes, and other cerebral lesions.

\section{SUMMARY}

Two versions of a new turning-bed called the Egerton/Stoke Mandeville turning-bed have been devised: one allows only turning from the supine to a 70-degree lateral position; the other allows both turning as well as head-up and head-down tilting to 14 degrees. The beds are electrically operated by motors fixed to either side of the bed. thus facilitating greatly the management of severely ill patients, in particular those with paralysis occurring after spinal-cord injury or disease. Postural reduction of severe fracture-dislocations can be successfully maintained during turning and the development of pressure sores prevented. The turning-bed can also be usefully employed in the management of other severe disabilities.

A new head-traction unit has been devised for the treatment of traumatic tetraplegics resulting from fracture-dislocation of the cervical spine. This device allows turning of the patient from side to side without discontinuation of the skull-traction cord; the head will remain in line with the axis through the length of the body, and correct traction be maintained during the process of turning.

REFERENCES

Guttmann, L. (1956). Practitioner, 176, 157. (1965). Paraplegia, 3, 193.

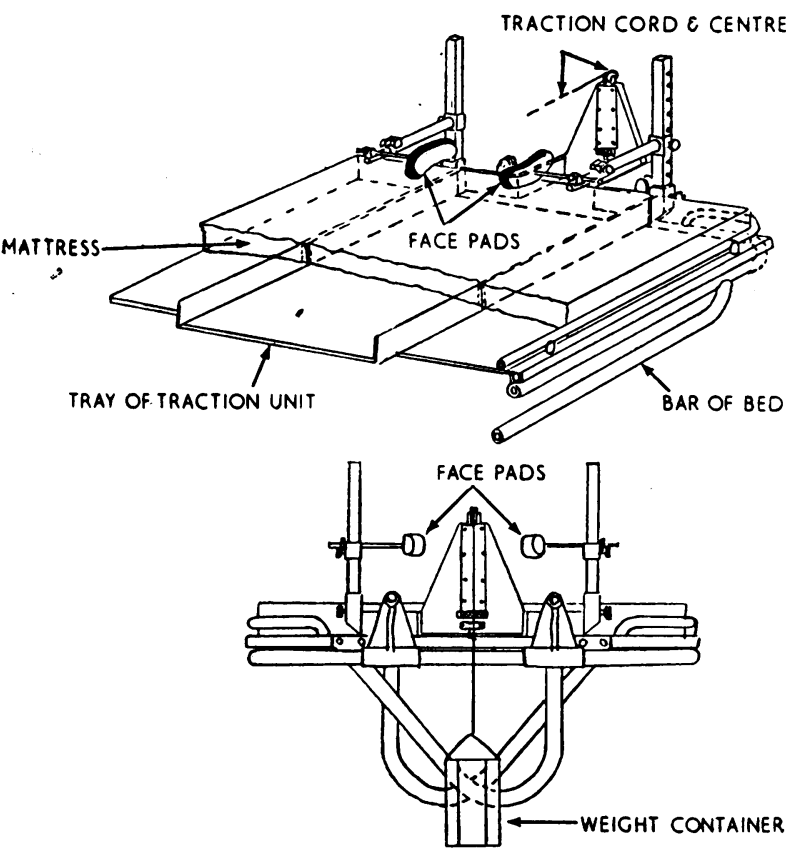

Fig. 4.-Guttmann head-traction unit.

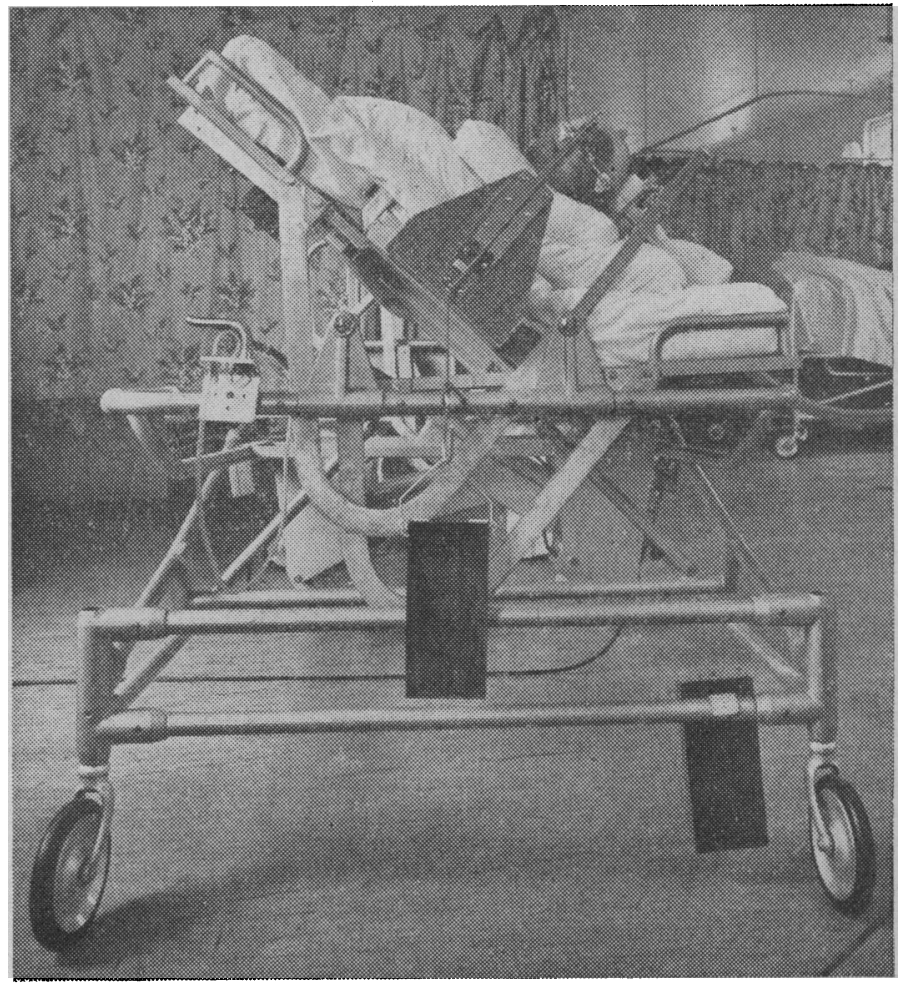

FIG. 5 